\title{
A $\beta$-SiC MOSFET Monte Carlo Simulator Including Inversion Layer Quantization
}

\author{
F. GÁMIZ, J. B. ROLDÁN * and J. A. LÓPEZ-VILLANUEVA \\ Departamento de Electrónica y Tecnología de Computadores, Universidad de Granada, Facultad de Ciencias, \\ Avd. Fuentenueva s/n, 18071 Granada (Spain)
}

\begin{abstract}
Electron transport properties in $\mathrm{SiC}$ quantized inversion layers have been studied by means of a Monte Carlo procedure. It has been observed that the contribution of polaroptical phonon scattering produces a significant influence of the effective-electric field on the high longitudinal field transport regime, this being the main difference of SiC with respect to standard $\mathrm{Si}$ inversion layers. The energy- and momentum-relaxation times have been calculated and the results suggest that electron velocity overshoot effects are less important than in Si MOSFETs. The electron mobility is not very different from their silicon counterparts, but the saturation velocity is higher.
\end{abstract}

Keywords: Silicon carbide, inversion layer, high field transport properties, electron mobility, polaroptical phonon scattering

\section{INTRODUCTION}

Cubic silicon carbide $(\beta$-SiC) is thought to be a material well-suited for electronic devices operating under harsh conditions such as high temperature, high power, and high frequency due to its high saturated drift velocity, wide band gap, and high thermal conductivity [1-2]. The fabrication of high temperature metal-oxide-semiconductor field-effect transistors (MOSFETs), junction fieldeffect transistors, and metal-semiconductor fieldeffect transistors has already been demonstrated [3].
As a consequence of this interest, theoretical analyses, and simulations on the electronic properties of silicon carbide have begun to appear in the last few years. However, up to now, numerical simulations related to the electron transport properties of silicon carbide inversion layers have not considered electron quantization. The wide experience obtained in silicon and other semiconductor material inversion layers over many years suggests that for a complete evaluation of the performance potential of silicon carbide MOSFETs, a detailed analysis of the transport properties of quantized silicon carbide inversion layers is essential.

\footnotetext{
*Corresponding author: Tel.: 34-58-246145. Fax. 34-58-2432300. E-mail: paco@gcd.ugr.es or juamba@gcd. ugr.es.
} 


\section{MONTE CARLO SIMULATOR}

Electron transport properties such as electron velocity and mobility at room and higher temperatures were studied by using a Monte Carlo simulator we have developed $[4,5]$. The position of the subband minima and the electron concentration of each of them were obtained by the self-consistent solution of the Poisson and Schroedinger equations for each value of the effectivetransverse-electric field. A non-parabolic band model was assumed taking $\alpha=0.323 \mathrm{eV}^{-1}$ [2], which limits our study to low-electron energies (below $1 \mathrm{eV}$ ). We have allowed the electron to travel in six subbands and to move between them. Acoustic, intervalley-optical and polar-optical phonons, surface-roughness and Coulomb scattering due to both bulk impurities and interfacetrapped and oxide-trapped charge have been taken into account.

\section{RESULTS}

We have simulated a SiC MOSFET with an oxide thickness of $150 \AA$ and a substrate impurity concentration $\mathrm{N}_{\mathrm{A}}=1 \times 10^{16} \mathrm{~cm}^{-3}$. We have paid special attention to the role that polar-optical phonon scattering plays in the total scattering rates. We have studied the ratio $R$ between the total scattering rates with and without the inclusion of polar-optical phonon scattering mechanisms. It has been plotted in Figure 1 for the lowest energy subbands versus electron kinetic energy. As can be observed, the lower the effective-electric field the higher the ratio $R$ for a wide range of electron kinetic energy. Therefore, the energy relaxation times are dominated by polar-optical phonon scattering at low effective-electric fields.

We have simulated long-channel $\beta$-SiC MOSFETs to obtain velocity curves versus longitudinal-electric field (Fig. 2). As can be seen, the drift velocity curves reach a maximum and then tend to decrease as the drift-electric field increases. There is a strong dependence of the velocity curves on the

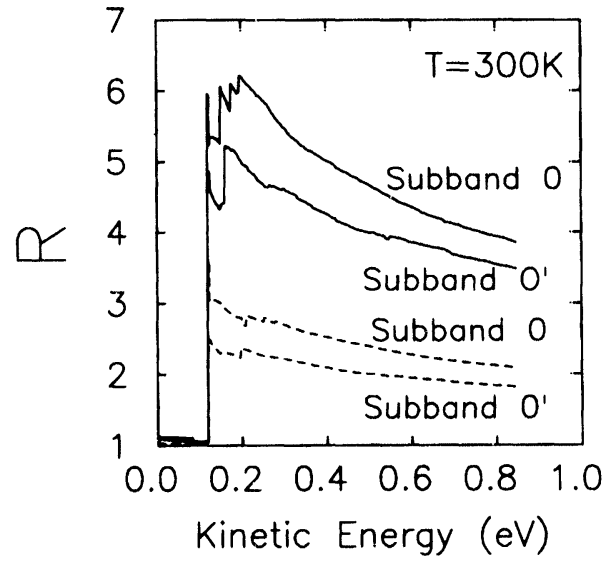

FIGURE 1 Ratio $R$ between the total scattering rates taking and not taking into account polar-optical phonon scattering for the first primed $(0)$ and unprimed $\left(0^{\prime}\right)$ subbands at $T=300 \mathrm{~K}$ for two different effective-electric fields: $E_{\mathrm{EFF}}=10^{5} \mathrm{~V} / \mathrm{cm}$ (solid line) and $E_{\mathrm{EFF}}=8 \times 10^{5} \mathrm{~V} / \mathrm{cm}$ (dashed line).

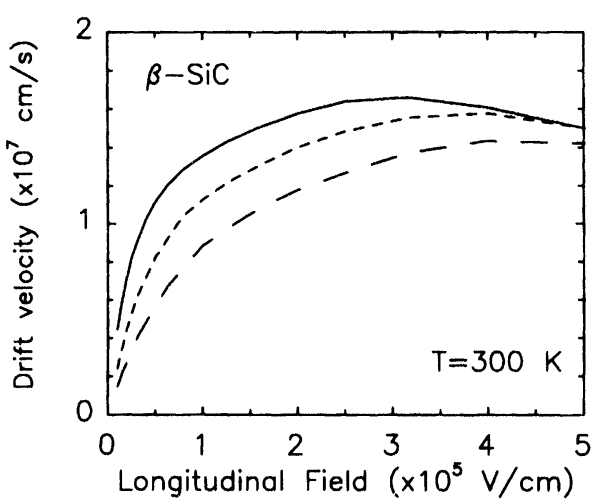

FIGURE 2 Homogeneous steady-state electron drift velocity curves in $\beta$-SiC at room temperature for different transverseeffective fields. (solid: $E_{\mathrm{EFF}}=1 \times 10^{5} \mathrm{~V} / \mathrm{cm}$, short dashed: $E_{\mathrm{EFF}}=5 \times 10^{5} \mathrm{~V} / \mathrm{cm}$, long dashed: $E_{\mathrm{EFF}}=1 \times 10^{6} \mathrm{~V} / \mathrm{cm}$ ) for $T=300 \mathrm{~K}$.

effective-electric field, this fact is connected with the results of Figure 1 (the lower the transverse field the higher the saturation velocity). The saturation velocity is higher than in silicon, which is one of the main reasons explaining the potential importance of silicon carbide as an electron device material, in addition to its wide band gap. Unfortunately there are no available experimental data to compare with our simulation data.

Making use of the steady-state velocity and energy plots we have calculated (Fig. 3) the energy- 


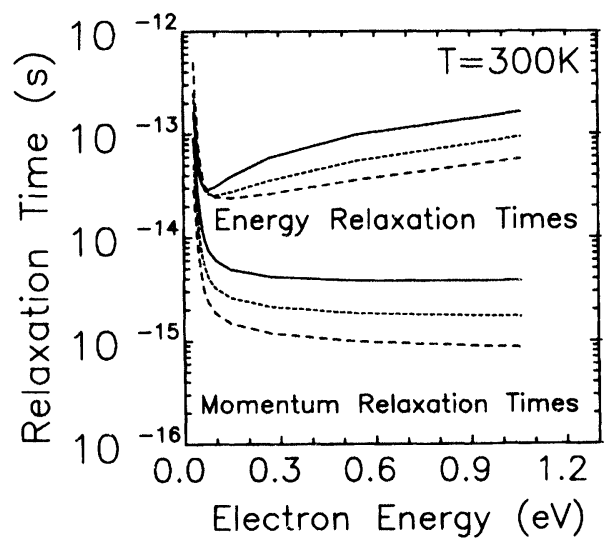

FIGURE 3 Momentum- and energy-relaxation times versus electron energy for a $\beta$-SiC MOSFET at $T=300 \mathrm{~K}$ (solid line) $E_{\mathrm{EFF}}=10^{5} \mathrm{~V} / \mathrm{cm}$, (short dashed line) $\mathrm{E}_{\mathrm{EFF}}=6.7 \times 10^{5} \mathrm{~V} / \mathrm{cm}$, (long dashed line) $E_{\mathrm{EFF}}=10^{6} \mathrm{~V} / \mathrm{cm}$.

and momentum-relaxation times at room temperature by means of the analytical expressions given in Ref. 6. This calculation has been done by using an average effective mass taking into consideration the fraction of electrons in the primed and unprimed subbands for each effective field. The $\mathrm{SiC}$ relaxation times are approximately one order of magnitude lower than the silicon ones, implying that the electron-velocity-overshoot effects are going to be less important than in silicon MOSFETs. At this point, it is worth highlighting the importance of the effective-electric field on the high longitudinal-electric-field transport properties in SiC MOSFETs. There exists a strong dependence of the saturation velocity (homogeneous transport in the channel) and of the relaxation times (inhomogeneous transport in the channel, velocity overshoot) on the effectiveelectric field, which is more important than in standard silicon MOSFETs.

We have simulated and plotted in Figure 4 the mobility curves versus the transverse-effective field at room temperature assuming (a) only phonon scattering (solid line), (b) phonon and surfaceroughness scattering (solid line and closed squares), (c) Coulomb scattering due only to substrate doping impurities (solid line and trian-

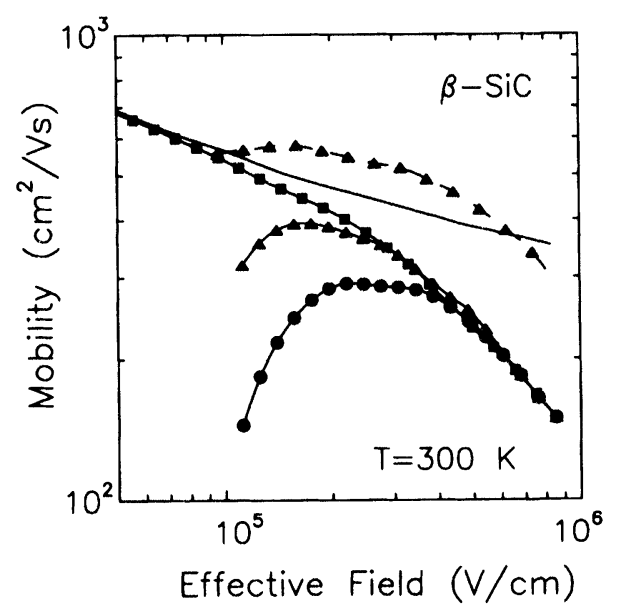

FIGURE 4 Electron mobility curves in $\beta$-SiC versus transverse effective field at room temperature: (a) only phonon scattering (solid line), (b) phonon and surface-roughness scattering (solid line and full squares), (c) Coulomb scattering due only to substrate doping impurities (solid line and full triangles), and (d) assuming, in addition to what is included in (c), a typical interface charged layer of $\mathrm{N}_{\mathrm{it}}=1 \times 10^{11} \mathrm{~cm}^{-2}$ (solid line and full circles). Electron mobility curve in $\mathrm{Si}$ (dashed line and full triangles).

gles), and (d) assuming, in addition, a typical interface charged layer of $\mathrm{N}_{\mathrm{it}}=1 \times 10^{11} \mathrm{~cm}^{-2}$ (closed circles). A mobility curve for a $\mathrm{Si}$ MOSFET corresponding to case (c) is included in dashed line and triangles. When only phonon scattering is taken into account, the electron mobility at low transverse-electric fields approaches the $\mathrm{SiC}$ bulk-mobility value $\left(1000 \mathrm{~cm}^{2} /\right.$ Vs). The reduction of the average distance between the inversion charge and the oxide interface increases surface-roughness and Coulomb scattering. In this respect, surface-roughness, Coulomb, and phonon-scattering probabilities (the last one is higher in $\mathrm{SiC}$ due to the additional contribution of polar-optical phonons), which are the three main scattering mechanisms that limit electron mobility in the channel of a MOSFET, are higher in SiC than in Si. It is clear that SiC MOSFETs are by no means candidates to substitute conventional $\mathrm{Si}$ ones at room temperature. However, at high temperature, where Si MOSFETs can no operate well, $\mathrm{SiC}$ devices play their major role. 


\section{CONCLUSIONS}

Electron transport properties have been evaluated in $\mathrm{SiC}$ quantized inversion layers. It has been shown that the influence of the effective-electric field on the high longitudinal field transport regime is significant, mainly due to the contribution of polar-optical phonon scattering which is essential at low effective fields for low and room temperatures. The electron-velocity-overshoot effects are less important than in Si MOSFETs. The electron mobility is not too different from their silicon counterparts, but the saturation velocity is nevertheless higher. So, this transport feature, in addition to a wide band gap, and high thermal conductivity, make $\mathrm{SiC}$ a promising candidate for high-temperature/high-power applications.

\section{References}

[1] Ivanov, P. A. and Chelnokov, V. E. (1992). "Recent development in SiC single-crystal electronics", Semicond. Sci. Technol., 7, 863-880.

[2] Tsukioka, K., Vasileska, D. and Ferry, K. (1993). "An ensemble Monte Carlo study of high-field transport in $\beta$ SiC", Physica B., 185, 467

[3] Palmour, J. W., Kong, H. S. and Davis, R. F. (1988). "Characterization of device parameters in high-temperature metal-oxide-semiconductor field-effect transistors in $\beta$-SiC thin films", J. Appl. Phys., 64, 2168-2177.

[4] Gámiz, F., Roldán, J. B., López-Villanueva, J. A. and Carceller, J. E. (1996). "Electron velocity saturation in quantized silicon carbide inversion layers", Appl. Phys. Lett., 69, 2219-2221.

[5] Roldán, J. B., Gámiz, F., López-Villanueva, J. A. and Cartujo, P. (1997). "Electron transport properties of quantized silicon carbide inversion layers", J. Electr. materials, 26, 203-207.

[6] Bordelon, T. J., Wang, X. L., Maziar, C. M. and Tasch, A. F. (1991). "An evaluation of energy transport models for silicon device simulation", Solid-State Electron., 34, 617.

\section{Authors' Biographies}

Juan B. Roldán graduated with a degree in physics in 1993, and received the Ph.D. in 1997 from the
University of Granada. Since 1993 he has been working on the MOS device physics including 2D transport, non-local effects and Monte Carlo simulations. Current interests are also related to $\mathrm{SiGe}$ and $\mathrm{SiC}$ devices. He is a Teaching Assistant at the University of Granada.

Francisco Gámiz graduated with a degree in physics in 1991, and received the Ph.D. in 1994 from the University of Granada. Since 1991 he has been working on the characterization of scattering mechanisms and their influence on the transport properties of charge carriers in semiconductor heterostructures. His current research interest includes the effects of many-carriers on the electron mobility and the interpretation of the influence of high longitudinal electric fields have on MOS transistors. Current interest are also related to $\mathrm{SiGe}$ and $\mathrm{SiC}$, and SOI devices, and quantum transport. $\mathrm{He}$ has coauthored several papers in all these subjects. $\mathrm{He}$ is an Associate Professor at the University of Granada.

Juan A. López-Villanueva graduated in 1984, Ph.D. in 1990 (University of Granada) with a thesis on the degradation of MOS structures by Fowler-Nordheim tunneling. Since 1985 he has been working on deep-level characterization and, mainly, MOS device physics, including Fowler-Nordheim and direct tunneling, quantum effects, 2D transport, effects of nonparabolicity, scattering mechanisms and Monte Carlo simulation of charge transport. He has coauthored several papers in all these subjects. His current research interest includes, simulation and modelling of electron devices. His educational activities also include analog systems for electronic instrumentation and power electronics. $\mathrm{He}$ is an Associate Professor at the University of Granada. 

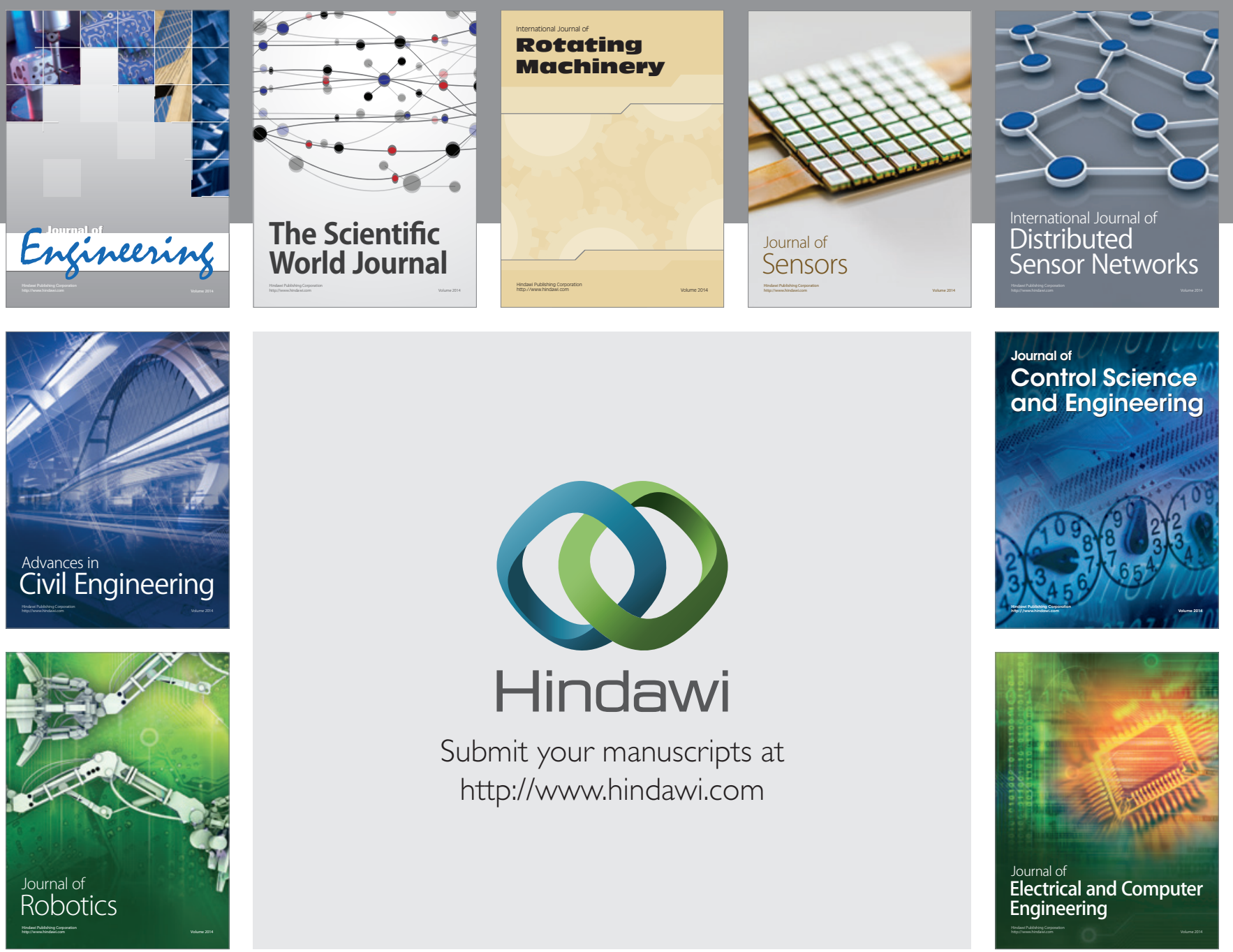

Submit your manuscripts at

http://www.hindawi.com
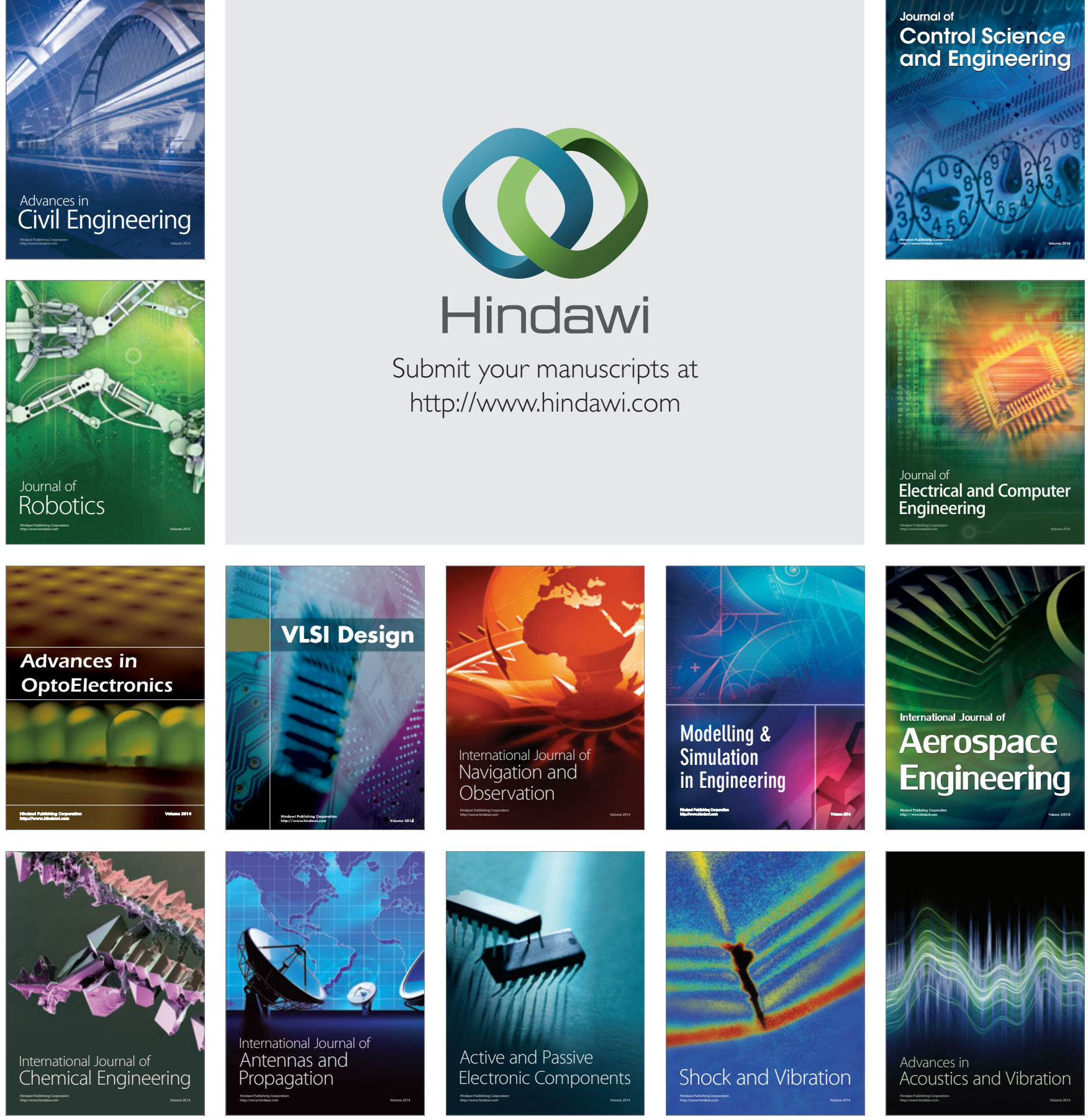